OPEN ACCESS

Edited by:

Gjumrakch Aliev,

GALLY International Biomedical

Research, United States

Reviewed by:

Junhong Zhou,

Harvard Medical School,

United States

Daniel Keeser,

Ludwig Maximilian University

of Munich, Germany

*Correspondence:

Daria Antonenko

daria.antonenko@uni-greifswald.de

Agnes Flöel

agnes.floeel@med.uni-greifswald.de

†These authors have contributed equally to this work

Received: 17 May 2019 Accepted: 16 July 2019

Published: 16 August 2019

Citation:

Antonenko D, Thams F, Uhrich J, Dix A, Thurm F, Li S-C, Grittner U and

Flöel A (2019) Effects of a

Multi-Session Cognitive Training Combined With Brain Stimulation (TrainStim-Cog) on Age-Associated Cognitive Decline - Study Protocol for a Randomized Controlled Phase

Illb (Monocenter) Trial.

Front. Aging Neurosci. 11:200.

doi: 10.3389/fnagi.2019.00200

\section{Effects of a Multi-Session Cognitive Training Combined With Brain Stimulation (TrainStim-Cog) on Age-Associated Cognitive Decline - Study Protocol for a Randomized Controlled Phase Ilb (Monocenter) Trial}

Daria Antonenko ${ }^{1 * t}$, Friederike Thams ${ }^{1+}$, Jessica Uhrich ${ }^{1}$, Annika Dix ${ }^{2}$, Franka Thurm ${ }^{2}$, Shu-Chen Li ${ }^{2}$, Ulrike Grittner ${ }^{3,4}$ and Agnes Flöel ${ }^{1,5 *}$

\footnotetext{
'Department of Neurology, Universitätsmedizin Greifswald, Greifswald, Germany, ${ }^{2}$ Chair of Lifespan Developmental Neuroscience, Faculty of Psychology, TU Dresden, Dresden, Germany, ${ }^{3}$ Berlin Institute of Health (BIH), Berlin, Germany, ${ }^{4}$ Charité - Universitätsmedizin Berlin, Corporate Member of Freie Universität Berlin, Humboldt-Universität zu Berlin, and Berlin Institute of Health, Institute of Biometry and Clinical Epidemiology, Berlin, Germany, ${ }^{5}$ German Centre for Neurodegenerative Diseases (DZNE) Standort Greifswald, Greifswald, Germany
}

Background: With increasing aging populations worldwide, developing interventions against age-associated cognitive decline is particularly important. Evidence suggests that combination of brain stimulation with cognitive training intervention may enhance training effects in terms of performance gain or transfer to untrained domains. This protocol describes a Phase Ilb clinical trial that investigates the intervention effects of training combined with brain stimulation in older adults.

Methods: The TrainStim-Cog study is a monocentric, randomized, single-blind, placebo-controlled intervention. The study will investigate cognitive training with concurrent anodal transcranial direct current stimulation (tDCS) over the left dorsolateral prefrontal cortex (target intervention) compared to cognitive training with sham stimulation (control intervention) over nine sessions in 3 weeks, consisting of a letter updating task, and a three-stage Markov decision-making task. Fifty-six older adults will be recruited from the general population. Baseline assessment will be performed including neuropsychological screening and performance on training tasks. Participants will be allocated to one of the two study arms using block-wise randomization stratified by age and baseline performance with a 1:1 allocation ratio. Primary outcome is performance in the letter updating task after training under anodal tDCS compared to sham stimulation. Secondary outcomes include performance changes in the decisionmaking task and transfer tasks, as well as brain structure and functional networks assessed by structural, and functional magnetic resonance imaging (MRI) that are acquired pre- and post-intervention. 
Significance: The main aim of the TrainStim-Cog study is to provide evidence for behavioral and neuronal effects of tDCS-accompanied cognitive training and to elucidate the underlying mechanisms in older adults. Our findings will contribute toward developing efficient interventions for age-associated cognitive decline.

Trial registration: This trial was retrospectively registered at Clinicaltrials.gov Identifier: NCT03838211 at February 12, 2019, https://clinicaltrials.gov/ct2/show/NCT03838211.

Protocol version: Based on BB 004/18 version 1.2 (May 17, 2019).

Keywords: transcranial direct current stimulation, aging, cognitive training, working memory, decision-making, spatial learning, transfer

\section{INTRODUCTION}

Given the worldwide increase of the proportion of older adults, the development of interventions against age-related cognitive declines are of great scientific and public interest (United Nations, 2015). Cognitive fitness and preserved everyday life abilities in advanced age is considered one of the most desirable conditions for individual well-being (Woods et al., 2013; Yam and Marsiske, 2013; Yam et al., 2014).

The combination of brain stimulation and multi-session cognitive training may counteract and delay the onset of agerelated impairments (Mameli et al., 2014; Perceval et al., 2016). Concurrent application of transcranial direct current stimulation (tDCS) over relevant brain regions during task practice has the potential to improve task performance and induce sustained effects (Kuo and Nitsche, 2012; Mameli et al., 2014; Woods et al., 2016; Au et al., 2017; Berryhill, 2017; Antonenko et al., 2018). Studies that applied anodal tDCS over frontal brain regions during working memory practice in healthy older adults have shown maintained benefits for trained and untrained visuospatial memory abilities and everyday life-relevant tasks (Jones et al., 2015; Stephens and Berryhill, 2016). Even if immediately measurable effects are absent (Nilsson et al., 2017), this intervention holds promise to exert long-lasting benefits by increasing neural plasticity (Jones et al., 2015). Therefore, clinical trials in older adults have to assess multiple cognitive outcomes, including those relevant for activities of daily living, and cover multiple time points, including long-term follow-up-assessment.

Modulation of brain network functioning has been suggested as potential underlying mechanism of behavioral improvement through tDCS (Meinzer et al., 2012; Hunter et al., 2013; Elmasry et al., 2015). Effects of tDCS-assisted multi-session cognitive training on structural and functional brain plasticity, and their relation to changes in cognitive scores, are however, largely unknown. In a recent study, we found increased functional coupling in the default mode network in older adults that was associated with immediate episodic memory training gains (Antonenko et al., 2018). Individual microstructural properties of white matter pathways may mediate neural and behavioral plasticity induced by tDCS (Lindenberg et al., 2013; Bachtiar et al., 2018). Thus, augmented neural network functioning may result in functional effects.

However, evidence for cognitive benefits, maintenance, and transfer of practice effects to untrained abilities is still mixed with high variability among older adults and research studies (Horvath et al., 2015; Antonenko et al., 2017; Kulzow et al., 2017; Nilsson et al., 2017; Passow et al., 2017). To be able to draw firm conclusions about the efficiency of these combined interventions, well-controlled randomized clinical trials in older adults with age-associated cognitive decline are required. In addition, investigations with cognitively impaired older adults can be based on results from studies in healthy adults (Perceval et al., 2016; Summers et al., 2016).

In the TrainStim-Cog study, we will conduct a randomized clinical trial implementing a multi-session working memory training in older adults $(n=56)$. Anodal tDCS over the dorsolateral prefrontal cortex (DLPFC) will be applied during task practice in half of the participants while the other half will receive sham stimulation. Stratified randomization will assure that the two groups are comparable regarding their age and baseline performance on the trained memory updating task. Effects on performance in trained and non-trained abilities as well as on brain function and structure will be assessed at multiple time-points. The current protocol describes the design and methods implemented in the TrainStim-Cog study. This protocol was prepared in accordance with the SPIRIT guidelines (Chan et al., 2013a,b).

\section{METHODS: PARTICIPANTS, INTERVENTION, AND OUTCOMES}

\section{Design and Setting}

This is a monocentric, randomized, single-blind, placebocontrolled study, including a nine-session cognitive training intervention in 3 weeks, accompanied by anodal tDCS over the left DLPFC compared to sham tDCS. Subjects will participate altogether in 14 sessions with pre- and post-intervention assessments that include magnetic resonance imaging (MRI), taking place at University Medicine Greifswald. Two follow-up sessions (at one and 7 months post-training) will be performed to also assess possible maintenance effects. A flow chart of the study is shown in Figure 1.

\section{Eligibility Criteria}

Before randomization, participants eligible for the study must meet all following criteria: 


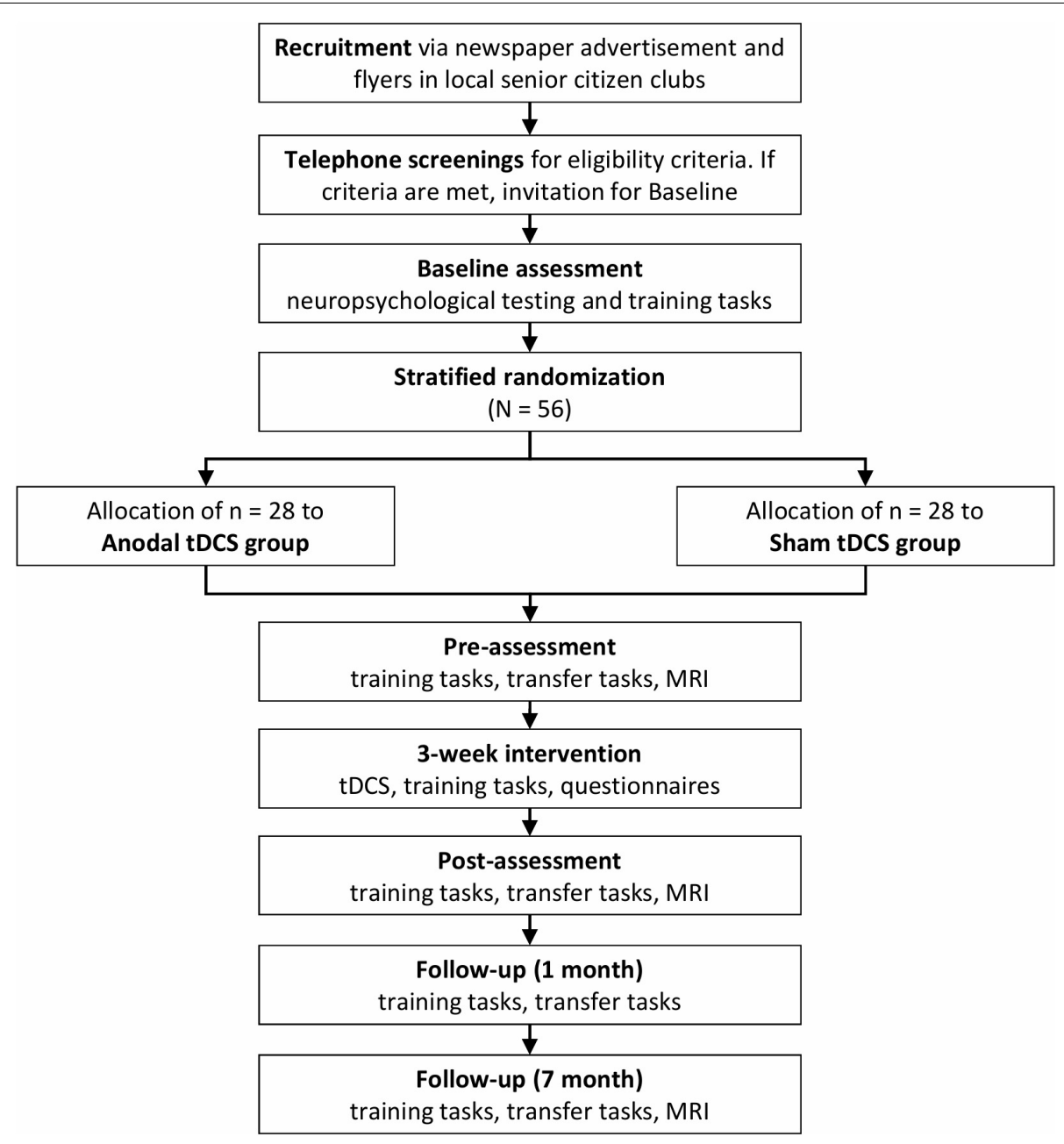

FIGURE 1 | TrainStim-Cog study flowchart. tDCS, transcranial direct current stimulation; MRI, magnetic resonance imaging.

(1) Age: $65-80$ years.

(2) Right-handedness.

(3) Performance in neuropsychological screening at baseline within normal range (defined as performance of each subtest within -1.5 standard deviations (SD) from the normative sample's mean).

In case one or more of the following criteria are present at randomization, potential participants will be excluded:

(1) Mild cognitive impairment (MCI), subjective cognitive decline (SCD), or dementia (participants reporting decline in cognitive functions or performing below $-1.5 \mathrm{SD}$ in any neuropsychological screening subtest will be excluded).

(2) Other neurodegenerative neurological disorders; epilepsy or history of seizures; close relatives with epilepsy or history of seizures; previous stroke.

(3) Severe and untreated medical conditions that preclude participation in the training, as determined by responsible physician.
(4) History of severe alcoholism or use of drugs.

(5) Severe psychiatric disorders such as depression (if not in remission) or psychosis.

(6) Contraindication to MRI (claustrophobia, metallic implants, ferromagnetic metals in the body, disorders of thermoregulation, and pregnant women) and tDCS (cf. Antal et al., 2017).

If all eligibility criteria are met and participants provide written informed consent, they will be included in the study sample.

\section{Intervention}

In each of the nine training sessions, participants will receive either anodal or sham tDCS while performing two cognitive training tasks, which are displayed in Figure 2A. Before starting the two training tasks, tDCS set-up will be mounted and stimulation will be started.

First, participants are presented with a letter updating task (cf. Dahlin et al., 2008) on a tablet computer, which will train 
A Training tasks

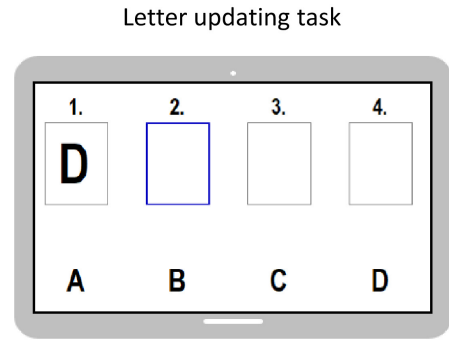

$\sim 12 \min$

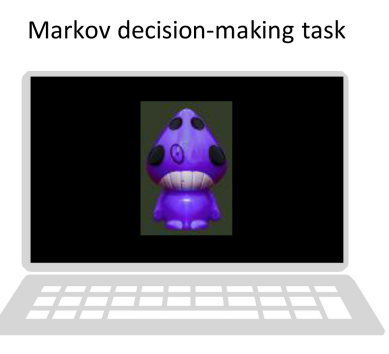

$\sim 15 \min$

B Transfer tasks

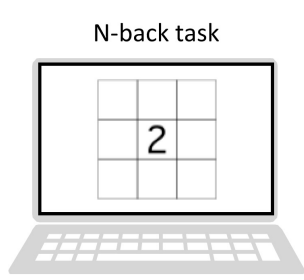

$\sim 10 \mathrm{~min}$
AVLT

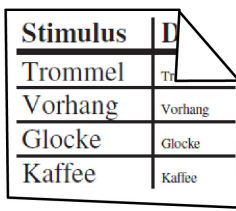

$\sim 10$ min
WMT2

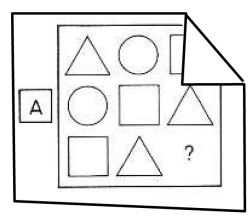

$30 \mathrm{~min}$

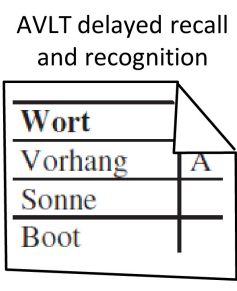

$\sim 10 \mathrm{~min}$

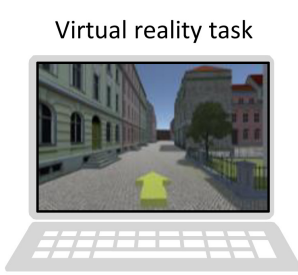

$\sim 45 \min$

FIGURE 2 | Task overview. (A) Training tasks performed at each session. (B) Transfer tasks performed at pre-, post-, and follow-up-assessments. AVLT, auditory verbal learning test (Helmstaedter et al., 2001). WMT-2, Wiener matrices test (Formann et al., 2011).

updating of information stored in working memory. Lists of letters A to D (with lengths of $5,7,9,11,13$, or 15 letters; three times each; total of 18 lists) will be presented in random order, one letter at a time (presentation duration: $2000 \mathrm{~ms}$, ISI $500 \mathrm{~ms}$ ). After each list, participants will be asked to recall the last four letters that were presented.

The second training task will be a three-stage Markov decision-making task (Eppinger et al., 2015; cf. Tanaka et al., 2016; Wittkuhn et al., 2018), presented on a laptop computer. Participants will be instructed to choose between two actions, i.e., pressure of left or right key, which results in an actionrelated reward (monetary gain or loss). Participants will have to learn to choose the optimal sequence of action to maximize overall gains and minimize overall losses. Markov probability refers to the fact that a decision at a given stage not only determines reward, but also the transition into the next stage (out of three stages) of stimulus choice decision. Therefore, choosing the optimal sequence of action will result in continuously transitioning through all three stages. The task will consist of two different learning conditions. In the immediate reward condition, the optimal action choice will consistently be rewarded with a gain $(+€ 0.05)$. Choosing the other alternative will be punished $(-€ 0.05)$, meaning that action-outcome associations will be equal for all three stages. In the delayed reward condition, optimal action choice will be associated with a small loss $(-€ 0.05)$ in the first two stages and a large gain $(+€ 0.25)$ in the third stage. Suboptimal action choice however, will be associated with a small gain $(+€ 0.05)$ in the first two stages and a large loss $(-€ 0.25)$ in the third stage. Thus, action-outcome associations will vary over the three stages in the delayed reward condition.
Transcranial direct current stimulation will be administered via a battery-operated stimulator (Neuroelectrics Starstim Home Research Kit, Barcelona, Spain). Direct current will be delivered with $1 \mathrm{~mA}$ intensity via two round saline-soaked synthetic sponge electrodes (5 cm diameter), connected to the stimulator and mounted in a neoprene head cap using the 10-20 EEG-system grid. Stimulation will consist of $20 \mathrm{~min}$ of continuous stimulation with ten additional seconds of ramping at the beginning and end of stimulation, respectively. The anodal electrode will be placed over left DLPFC (F3). Placement of the cathodal electrode is the contralateral supraorbital cortex (Fp2). In the sham tDCS group, the same electrode montage and ramp-time will be used, but current will only be applied for $30 \mathrm{~s}$ to elicit the typical tingling sensation of stimulation on the scalp and to blind participants regarding the stimulation condition (see section "Blinding"). Stimulation will be started simultaneously with the letter updating task and run during its entire length and approximately the first half of the Markov task. After every third session, participants will fill out an adverse-events questionnaire (Antal et al., 2017). Participants will be instructed to avoid excessive alcohol consumption or smoking on the day of the study, to adhere to their usual sleep duration, and to avoid drinking caffeine $90 \mathrm{~min}$ prior to the training sessions.

\section{Outcome Measures}

Outcome measures for the training tasks will be acquired at each session. At pre-, post- and follow-up-assessments, additional outcome measures targeting transfer effects (see Figure 2B for an overview), will be assessed. All measures and time points of acquisition are listed 
in Table 1. Each outcome measure will be analyzed regarding potential differences between intervention groups (anodal vs. sham tDCS).

\section{Primary Outcomes}

Primary outcome measure will be working memory performance at post-assessment, operationalized by number of correctly recalled lists in the letter updating task.

\section{Secondary Outcomes}

Secondary outcome will be performance in decision-based learning at post-assessment, as measured by proportion of optimal actions in the Markov decision-making task.

Additionally, performance at follow-up-assessments will be analyzed for the main measures of both training tasks (number of correctly recalled lists in the letter updating task and proportion of optimal actions in the Markov decision-making task). Further, online and offline effects of the intervention will be assessed for the main measures of both training tasks. Online effects will be defined as within session performance changes, whereas changes in performance from the last trial of the previous visit to the first trial of the next visit will be considered as offline effects (Reis et al., 2009). The total direct effects of the intervention will be assessed by analyzing learning curves (change from first to last training session) for both training tasks measures.

Further secondary outcomes will be assessed at pre-, post-, and follow-up-assessments and include:

(a) Transfer tasks encompassing working memory performance, as assessed by performance in a numeric n-back task (\% correct, d-prime); episodic memory performance, as measured by performance in the German version of the auditory verbal learning test (Helmstaedter et al., 2001; Lezak et al., 2004) (total amount of words learned, number of recalled words at delayed recall), reasoning ability, as assessed by the Wiener matrices test (WMT-2) (Formann et al., 2011) (\% correct), spatial memory ability, as measured by a virtual reality maze task (Hartley et al., 2003) (number of items found on a previous encoded route). All transfer measures will be corrected for performance at pre-assessment.

(b) Structural and functional neural correlates (assessed at pre-, post-, and 7-months follow-up-assessments), as measured by structural and functional MRI.

\section{Exploratory Analyses}

Exploratory analyses will be conducted for more detailed outcomes of the two training tasks (e.g., outcomes dependent on list length in the letter updating task, parameters from a drift diffusion model for the Markov decision-making task). Additionally, measures of cognitive reserve such as education, baseline cognitive ability or neuropsychological status will be analyzed for identifying potential predictors of training task performance, and responsiveness to the intervention. Genetic polymorphisms such as ApoE, COMT and BDNF, derived from analysis of blood samples and related to cognition, will be included as potential modulators of response to tDCS (Fritsch et al., 2010).

\section{Participant Timeline}

Participants will adhere to 14 sessions with three additional MRI sessions, taking place at the University Medicine Greifswald. After completion of baseline assessment (V0), participants will successively be invited to start the training sessions (V2-V10), which will take place during three consecutive weeks on 3 days a week. Three days before and after training sessions, pre- and post-assessments (V1 and V11) will be conducted. Four weeks after post-assessment a first follow-up session (V12) will be administered; second follow-up (V13) will be 7 months after post-assessment. MRI will be measured directly before and after the training (V1 and V11) and at second follow-up (V13). An overview of all visits and outcome measures is provided in Table 1.

\section{Baseline Measures}

At baseline assessment (V0), participants will give written informed consent, and participate in a demographic interview. Depression screening and handedness questionnaire will be administered. Furthermore, performance in several cognitive domains will be tested (Table 1). Afterward, participants will perform the two training tasks as described above, except that at baseline, the letter updating task will consist of one practice trial with 4 lists and one actual trial with 15 lists (compared to one trial of 18 lists in the training). Baseline visit will take approximately $3 \mathrm{~h}$.

\section{Pre-, Post-, and Follow-Up-Assessments}

All four sessions will comprise the same procedure. Initially, self-reported well-being, quality, and duration of sleep as well as potential stressors $2 \mathrm{~h}$ prior to the session are assessed by the investigator in a semi-structured interview. After having performed the two training tasks, participants will accomplish several transfer tasks, testing multiple memory functions, and reasoning ability. The 7-months follow-up-assessment will provide the possibility for assessing the maintenance of training and transfer effects.

\section{Sample Size}

Based on recent studies in the field using multi-session application of anodal tDCS during cognitive training compared to training with sham tDCS (Park et al., 2014; Jones et al., 2015; Antonenko et al., 2018), we estimated an effect size of 0.85 . To demonstrate an effect in the primary outcome, 46 participants (23 per group) need to be included in the analysis with an independent $t$-test using a two-sided significance level of 0.05 and a power of $80 \%$. This conservative approach using a $t$-test was chosen, even though we intend to analyze the primary outcome conducting analysis of covariance (ANCOVA) models (Borm et al., 2007). Assuming a drop-out rate of about 20\% due to a high number of planned visits and considerably high demands put upon participants (e.g., performing challenging memory tasks and attending three sessions of 45 min MRI scans), 28 participants should be included in each tDCS group. 
TABLE 1 | TrainStim-Cog outcome measures.

\begin{tabular}{|c|c|c|c|c|c|c|c|c|}
\hline & & & \multicolumn{6}{|c|}{ Post-allocation } \\
\hline & & & Base line & Pre & T1-T9 (3 weeks) & Post (3 days) & FU (1 month) & FU (7 months) \\
\hline & & & $\sim 2 \mathrm{~h}$ & $\sim 3 \mathbf{h}$ & $\sim 1 \mathrm{~h}$ & $\sim 3 \mathbf{h}$ & $\sim 3 \mathbf{h}$ & $\sim 3 \mathbf{h}$ \\
\hline Time point & Measurement & Mode & vo & V1 & V2-V10 & V11 & V12 & V13 \\
\hline \multicolumn{9}{|l|}{ Enrollment } \\
\hline Eligibility screening & & Paper & $x$ & & & & & \\
\hline Informed consent & & Paper & $\times$ & & & & & \\
\hline \multirow{6}{*}{$\begin{array}{l}\text { Neuro } \\
\text { psycho-logical } \\
\text { screening }\end{array}$} & Demographic data & Paper & $\times$ & & & & & \\
\hline & $\begin{array}{l}\text { Geriatric depression scale } \\
\text { (Brink et al., 2013) }\end{array}$ & Paper & $x$ & & & & & \\
\hline & $\begin{array}{l}\text { Oldfield handedness inventory } \\
\text { (Oldfield, 1971) }\end{array}$ & Paper & $\times$ & & & & & \\
\hline & CERAD (memoryclinic.ch ${ }^{1}$ ) & Paper & $\times$ & & & & & \\
\hline & Digit span (Lezak et al., 2004) & Paper & $\times$ & & & & & \\
\hline & $\begin{array}{l}\text { Identical pictures (Lindenberger } \\
\text { and Baltes, 1997) } \\
\text { Spot-a-word (Lehrl, 1977) }\end{array}$ & Computer & $\times$ & & & & & \\
\hline \multicolumn{9}{|l|}{ Intervention } \\
\hline \multirow[t]{2}{*}{ Training tasks } & $\begin{array}{l}\text { Letter updating (Dahlin et al., } \\
\text { 2008) }\end{array}$ & Tablet-PC & $\times$ & $x$ & $x$ & $x$ & $x$ & $x$ \\
\hline & $\begin{array}{l}\text { Markov decision-making } \\
\text { (Eppinger et al., 2015; Wittkuhn } \\
\text { et al., 2018) }\end{array}$ & Computer & $\times$ & $x$ & $x$ & $x$ & $x$ & $x$ \\
\hline Brain stimulation & tDCS (anodal vs. sham) & Device & & & $x$ & & & \\
\hline \multirow[t]{3}{*}{ Questionnaires } & Self-reported well-being & Paper & & $x$ & $x$ & $x$ & $x$ & $x$ \\
\hline & PANAS (Watson et al., 1988) & Paper & & & $x$ & & & \\
\hline & $\begin{array}{l}\text { Adverse events questionnaire* } \\
\text { (Antal et al., 2017) }\end{array}$ & Paper & & & $x$ & & & \\
\hline \multicolumn{9}{|c|}{ Additional assessments } \\
\hline \multirow[t]{4}{*}{ Transfer tasks } & n-back & Computer & & $x$ & & $x$ & $x$ & $x$ \\
\hline & $\begin{array}{l}\text { AVLT (Helmstaedter et al., } \\
\text { 2001; Lezak et al., 2004) }\end{array}$ & Paper & & $x$ & & $x$ & $x$ & $x$ \\
\hline & $\begin{array}{l}\text { Wiener matrices test (Formann } \\
\text { et al., 2011) }\end{array}$ & Paper & & $x$ & & $x$ & $x$ & $x$ \\
\hline & $\begin{array}{l}\text { Virtual reality task (Hartley et al., } \\
\text { 2003) }\end{array}$ & Computer & & $x$ & & $x$ & $x$ & $x$ \\
\hline \multirow[t]{2}{*}{ Physical measures } & $\underline{\mathrm{MRI}}$ & & & $x$ & & $x$ & & $x$ \\
\hline & Blood draw & & \multicolumn{4}{|c|}{ Once at any of these sessions } & & \\
\hline
\end{tabular}

T1-T9, training 1-9; FU, follow-up-assessment; V0-V13, visits 0-13; CERAD, the consortium to establish a registry for Alzheimer's Disease, neuropsychological battery. PANAS, positive and negative affect schedule; AVLT, German version of the auditory verbal learning test; tDCS, transcranial direct current stimulation; MRI, magnetic

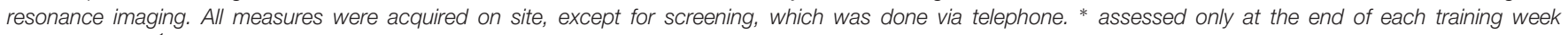
N4, 7, and 10). ${ }^{1}$ http://www.memoryclinic.ch/de/main-navigation/neuropsychologen/cerad-plus/.

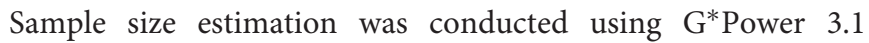
(Faul et al., 2007).

\section{Recruitment}

Participants will be recruited through advertisements in the local newspapers and distribution of flyers in local senior citizen clubs. Telephone screenings will be conducted with all potential participants and study information will be provided. Eligible candidates will be invited for baseline assessment. Following baseline assessment (V0) participants will be included if neuropsychological testing is unobtrusive.

\section{METHODS: ASSIGNMENT OF INTERVENTIONS}

We will first complete recruitment and baseline measurement of all subjects before allocating participants to the groups and starting the training sessions. Allocation will be performed by a researcher not involved in baseline assessments. Participants will be randomly allocated in 1:1 ratio to the experimental groups (anodal vs. sham tDCS), using age and initial performance in the letter updating task as strata. First, all participants that successfully completed telephone screening and baseline 
assessments will be divided into four groups by median split: (1) age $\leq$ median and LU performance $\leq$ median, (2) age $\leq$ median and LU performance $>$ median, (3) age $>$ median and $\mathrm{LU}$ performance $\leq$ median, and (4) age $>$ median and LU performance $>$ median. Second, equal numbers of participants of each group will be randomly assigned to anodal and sham tDCS group, respectively, using the blockrand package in $\mathrm{R}^{1,2,3}$. Allocation concealment will be ensured, since decision about in- or exclusion will already be made, before allocating participants to the groups.

\section{Blinding}

This is a single-blind trial, participants will be blind to the stimulation condition. In the sham tDCS group, current will be applied for $30 \mathrm{~s}$ to elicit the typical tingling sensation of stimulation on the scalp and to blind participants regarding the stimulation condition. Previous research showed that sham tDCS is a safe and valid method of blinding participants (Schlaug and Renga, 2008; Floel and Cohen, 2010). After the last training session, participants will be asked to state whether they believed they received anodal or sham tDCS. Note that in our single-blind design, implicit bias of the investigators during data collection cannot be completely excluded. However, to minimize bias in the analysis, data will be entered electronically, and will be analyzed, by a member of the research team blinded to the stimulation condition, according to the statistical analysis plan.

\section{METHODS: DATA COLLECTION, MANAGEMENT, AND ANALYSIS}

\section{Data Collection Methods}

Neuropsychological, behavioral and MRI data and blood samples will be collected from each participant. Study assessors will be thoroughly trained in administering the assessments. Time points of data collection are shown in Table 1.

\section{Neuropsychological and Behavioral Assessment}

Neuropsychological tests at baseline visit (V0) comprise paper-pencil as well as computer-based assessments. Geriatric Depression Scale (Brink et al., 2013) and the Edinburgh Handedness Inventory (Oldfield, 1971) will be administered. Performance in several cognitive domains will be tested with CERAD-Plus test battery ${ }^{4}$ (Morris et al., 1989), digit span test (Lezak et al., 2004), identical-pictures task (Lindenberger and Baltes, 1997), and spot-a-word task (Lehrl, 1977).

Training- and transfer tasks include paper-pencil and computer-based assessments. Detailed description of the two training tasks is provided in the interventions section. In

\footnotetext{
${ }^{1}$ http://www.R-project.org

${ }^{2}$ http://www.rstudio.com

${ }^{3}$ https://CRAN.R-project.org/package=blockrand

${ }^{4}$ http://www.memoryclinic.ch/de/main-navigation/neuropsychologen/ceradplus/
}

pre-, post- and follow-up sessions (V1, V11-V13), transfer tasks will be administered: Participants will perform a numeric n-back task (1 and 2 back) and the German version of the auditory verbal learning test (Helmstaedter et al., 2001; Lezak et al., 2004). In the $30 \mathrm{~min}$ interval to assess longterm memory, participants will perform the Wiener matrices test (WMT-2) (Formann et al., 2011). Then, a virtual reality navigation task will be presented (Hartley et al., 2003). Here, during encoding, participants will be instructed to memorize a route with several targets (e.g., butcher, doctor's office, and grocery store); during subsequent recall, the participants will be asked to navigate the shortest route to given targets.

\section{Magnetic Resonance Imaging}

Magnetic resonance imaging will be assessed at the Baltic Imaging Center (Center for Diagnostic Radiology and Neuroradiology, Universitätsmedizin Greifswald) with a 3 Tesla scanner (Siemens Verio) using a 32-channel head coil, 1 day prior to and 2 days as well as 7 months after training (see Table 2 for neuroimaging data acquisition parameters). A T1-weighted 3D sequence, a 3D FLAIR, a diffusion tensor imaging (DTI) and a resting-state fMRI sequence will be assessed. At the end of the MRI assessment, additional T1and T2-weighted structural images will be acquired with parameters optimized for computational modeling to calculate electric field distributions ${ }^{5}$ [(Windhoff et al., 2013; Thielscher et al., 2015), see Figure 3 for sample modeling analysis]. Detailed information about MRI sequences is provided in Table 2. Pipelines from MATLAB-based toolboxes such as SPM (Wellcome Department of Imaging Neuroscience, London, United Kingdom ${ }^{6}$ ), CONN toolbox ${ }^{7}$ (Whitfield-Gabrieli and Nieto-Castanon, 2012), or FSL (Analysis Group, FMRIB, Oxford, United Kingdom ${ }^{8}$ ) (Jenkinson et al., 2012) and Freesurfer ${ }^{9}$ will be used for analysis of structural and functional MRI data. To assess volume of cortical and subcortical gray matter, structural segmentation will be performed on T1-weighted scans (Dahlin et al., 2008; Filmer et al., 2019). White matter microstructure in main white matter tracts will be extracted from diffusion-weighted images using common tractography methods (Charlton et al., 2010; Metzler-Baddeley et al., 2011, 2017, Le Bihan and Johansen-Berg, 2012). Functional resting-state fMRI scans will be used to assess functional connectivity within and between large-scale networks that mediate cognitive functions (Darki and Klingberg, 2015; Antonenko et al., 2018, 2019a).

\section{Blood Draw}

At one of the sessions, a blood sample for conducting genetic analyses will be collected, preprocessed, and stored at the Neuroimmunology Lab of University Medicine Greifswald, using cryo-preservation method. Genetic polymorphisms

\footnotetext{
${ }^{5}$ http://simnibs.org

${ }^{6}$ www.fil.ion.ucl.ac.uk/spm/

${ }^{7}$ www.nitrc.org/projects/conn

${ }^{8}$ http://fsl.fmrib.ox.ac.uk/fsl/fslwiki/

${ }^{9}$ http://surfer.nmr.mgh.harvard.edu/
} 
TABLE 2 | Neuroimaging data acquisition parameters.

\begin{tabular}{|c|c|}
\hline Sequence & Main parameters \\
\hline $\begin{array}{l}\text { Resting-state } \\
\text { fMRI }\end{array}$ & $\begin{array}{l}\text { TR }=2000 \mathrm{~ms}, \mathrm{TE}=30 \mathrm{~ms} \text {, FOV } 192 \times 192 \mathrm{~mm}^{2}, 34 \text { slices, } \\
176 \text { volumes, descending acquisition, } 3.0 \times 3.0 \times 3.0 \mathrm{~mm}^{3} \text {, flip angle } 90^{\circ}\end{array}$ \\
\hline T1 MPRAGE & $\mathrm{TR}=2300 \mathrm{~ms}, \mathrm{TE}=2.96 \mathrm{~ms}, \mathrm{Tl}=900 \mathrm{~ms}, 192$ slices, $1.0 \times 1.0 \times 1.0 \mathrm{~mm}^{3}$, flip angle $9^{\circ}$ \\
\hline DTI & $\mathrm{TR}=11100 \mathrm{~ms}, \mathrm{TE}=107 \mathrm{~ms}, 70$ slices, $1.8 \times 1.8 \times 2.0 \mathrm{~mm}^{3}, 64$ directions $\left(b=1000 \mathrm{~s} / \mathrm{mm}^{2}\right)$ \\
\hline FLAIR & $\mathrm{TR}=5000 \mathrm{~ms}, \mathrm{TE}=388 \mathrm{~ms}, 160$ slices, $1.0 \times 1.0 \times 1.0 \mathrm{~mm}^{3}$ \\
\hline T1w & $\begin{array}{l}\mathrm{TR}=1690 \mathrm{~ms}, \mathrm{TE}=2.52 \mathrm{~ms}, \mathrm{TI}=900 \mathrm{~ms}, 176 \text { slices, } 1.0 \times 1.0 \times 1.0 \mathrm{~mm}^{3} \text {, flip angle } 9^{\circ} \text {, using } \\
\text { selective water excitation for fat suppression }\end{array}$ \\
\hline $\mathrm{T} 2 \mathrm{w}$ & $\mathrm{TR}=12770 \mathrm{~ms}, \mathrm{TE}=86.0 \mathrm{~ms}, 96$ slices, $1.0 \times 1.0 \times 1.0 \mathrm{~mm}^{3}$, flip angle $111^{\circ}$ \\
\hline
\end{tabular}

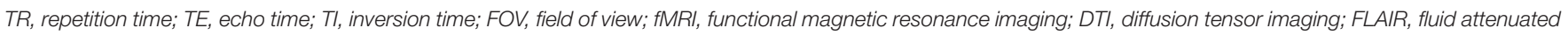
inversion recovery; MPRAGE, magnetization prepared rapid acquisition gradient echo.

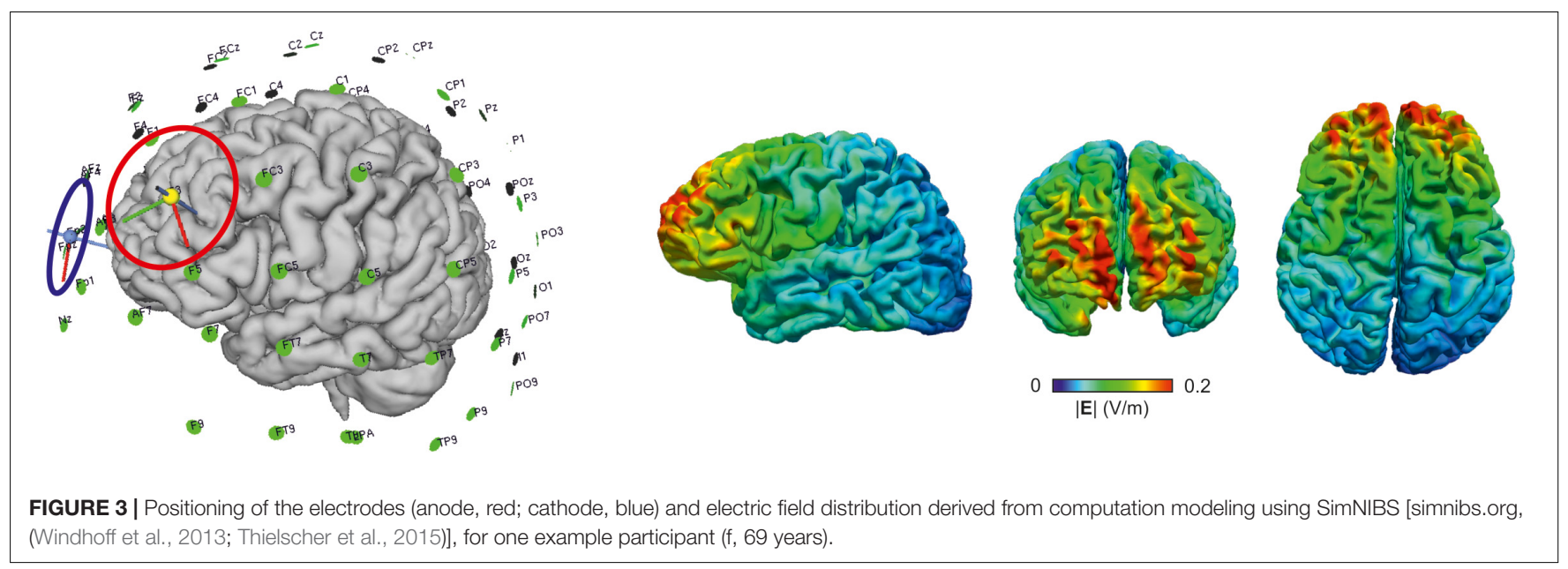

relevant for learning (such as ApoE, COMT, and BDNF) (Antal et al., 2010; Freitas et al., 2011; Witte et al., 2012; Plewnia et al., 2013) will be analyzed at the Department of Psychiatry, Psychotherapy and Psychosomatic, University Medicine, Halle/Saale, Germany.

\section{Retention and Adherence}

To maximize retention throughout the entire study period, participants will be provided with information about their appointments via telephone, and e-mail. A few days prior to starting the 3-weeks training period, participants will be contacted via telephone as a reminder of the upcoming appointments, and to clarify potential open questions. On pre-assessment, they will receive a printout of all study sessions. Additionally, time and date of the next session will be discussed at each visit. Participants will be encouraged to leave a message on the study site's 24/7 answering machine, if they have any concerns about not being able to attend a visit or wanting to change the time. They will then be contacted to discuss alternative scheduling. At the end of the study, participants receive a reasonable financial reimbursement (approximately $10 €$ per hour), the results of their neuropsychological screening, and structural MRI images on a compact disc. If complete adherence to the protocol is not possible, any effort to collect as much data as feasible from the participant will be made.

\section{Data Management and Monitoring}

All data collected will be pseudonymized. Files containing personal data of the participants will be saved with a password, solely known by staff involved in the project. Data acquired on paper will be entered electronically by research staff. To prevent erroneous entries, data will be entered by one person, and double-checked by another. Progress of data entry and checking procedures will be documented. Folders containing forms and questionnaires of each subject will be stored securely and sorted by participant ID number for easy access at each stage of the study. Forms containing names and personal data of participants will be stored separately in a lockable cabinet. Digital data, such as output files from computer-based tasks, will be stored on a secure file server directly after acquisition. Protocols of the tDCS setup of each participant and session will also be stored on the file server. MRI data will be pseudonymized before analysis. Following good scientific practice, data will be stored for at least 10 years.

\section{Adverse Events Monitoring}

Potential adverse events (AEs) occurring throughout the course of the study will be monitored via an adverse events questionnaire (Antal et al., 2017), administered after each third stimulation session. We will refrain from administering the AEs-questionnaire at each stimulation session, since this might unnecessarily draw the participant's attention to minor sensations 
during stimulation, and therefore serve as an unintentional distractor from the tasks. Generally, the risk of health damage due to anodal tDCS can be expected to be minimal. Known AEs with the study parameters $(20 \mathrm{~min}, 1 \mathrm{~mA})$ are tingling at the electrode sites, skin reddening under the electrode and, less frequently, a mild headache (Antal et al., 2017). Study assessors will be instructed to monitor AEs and serious AEs (SAEs) throughout the trial and document all detected AEs and SAEs. Participants will be informed at baseline visit about all possible risks and can withdraw consent at any time without providing reasons. In case an SAE occurs, the study physician will first make an assessment as to whether a causal relationship with the intervention is considered possible. If more than three of the enrolled participants suffer from SAEs that are likely to be associated with the intervention (as assessed by the study physician), the trial will be discontinued.

\section{Statistical Methods}

Statistical analyses of the primary and secondary outcome measures will be reported in detail in the statistical analysis plan, to be written and registered before data analyzes. Data from all participants included at randomization will be analyzed using intention to treat analyses (ITT). In case of missing values and under the assumption of missing at random we will use multiple imputation methods to estimate treatment effects. Additionally, a "per protocol" analysis will be conducted as sensitivity analysis, including only those participants, who completed all visits, and finished post-assessment. Focusing on the primary outcome, we will conduct an ANCOVA with the post-assessment working memory score (number of correctly recalled lists in the letter updating task) as dependent variable, stimulation group (anodal, sham) as factor, and working memory performance at pre-assessment as well as age as covariates. Secondary outcomes will be analyzed using similar statistical methods. For instance, an ANCOVA with performance in the Markov decision-making task at postassessment (proportion of optimal actions) as dependent variable, stimulation group (anodal, sham) as factor, and performance in the Markov decision-making task at preassessment as well as age as covariates will be conducted. We will furthermore analyze secondary outcome measures and their interactions, using linear mixed models with time-point (e.g., training days 1-9) as within-subject factor and stimulation group (anodal, sham) as between-subject factor. Changes in structural and functional neural parameters will be analyzed on whole-brain level, using general linear models, implemented in the analysis software. To assess brain-behavior associations, correlations between behavioral and neuronal parameters, will be calculated. In case of violation of requirements for parametric methods, appropriate non-parametric tests will be conducted. Data analysis will be conducted using IBM SPSS Statistics for Windows (IBM Corp., Armonk, NY, United States), MatLab (The Mathworks Inc., 2016), and R software ${ }^{10}$.

\footnotetext{
${ }^{10} \mathrm{https} / / /$ www.R-project.org
}

\section{ETHICS AND DISSEMINATION}

The study was approved by the ethics committee of the University Medicine Greifswald and will be conducted in accordance with the Helsinki Declaration. All data collected will by pseudonymized. Imaging data will be made publicly available to the general academic community at https://openneuro.org. Results of the study will be made accessible to scientific researchers and health care professionals via publications in peer reviewed journals and presentations at national and international conferences. Furthermore, the scientific and lay public can access the study results on the ClinicalTrials.gov website (Identifier: NCT03838211).

\section{DISCUSSION}

This randomized controlled trial will determine the impact of a multi-session memory training combined with anodal tDCS on trained and untrained functions as well as functional and structural neural parameters in cognitively intact older adults. In the target group, anodal tDCS ( $1 \mathrm{~mA}, 20 \mathrm{~min}$ ) will be applied over the DLPFC (with the cathode over the contralateral supraorbital cortex) during task practice while the control group will receive sham tDCS $(1 \mathrm{~mA}, 30 \mathrm{~s})$. Findings of this study will contribute to the understanding of immediate and delayed behavioral and neural effects, as well as the underlying mechanisms of tDCSplus-training effects in the aged brain.

Older adults constitute the target group of this trial as we aim to exert beneficial effects on age-related cognitive decline. We opted to elucidate the effects and underlying mechanisms in a population that serves as baseline for future trials targeting cognitively impaired older patients, for example MCI or dementia due to Alzheimer's disease (Perceval et al., 2016).

All participants will undergo extensive baseline assessment to obtain detailed participant characteristics and to assure cognitive functioning within age- and education-related norms. Stratified randomization will be performed to match anodal and sham groups for age and baseline performance in the training task. We argue that this is important to exclude chance age and baseline differences that impact tDCS-induced modulation and hamper the interpretation of effects (Martin et al., 2013; Antonenko et al., 2017, 2018). Furthermore, as tDCS may potentially exert longterm effects, even in the absence of immediate training gains, we will invite participants to two follow-up sessions one and 7 months after training (Jones et al., 2015; Berryhill, 2017).

We designed a cognitive training with two cognitive tasks that are mediated by the prefrontal cortex, vulnerable to the effects of aging, and well suited to examine effects of practice over multiple sessions in older adults (Dahlin et al., 2008; Eppinger et al., 2015; Wittkuhn et al., 2018). The primary task (letter updating) has shown good applicability and training-induced plasticity in older adults (Dahlin et al., 2008). To study near and far transfer of training, we chose tasks which measure working and episodic memory, reasoning and visuospatial abilities. Altogether, we aimed to design a comprehensive assessment of multiple task domains at multiple time points avoiding floor or ceiling 
effects, which is also not too long or too exhausting for older participants. This multimodal testing, including paperpencil, computer- and tablet-based tasks will not only provide reliable data on training and transfer effects, but also be attractive to the participants, assuring their compliance, and motivation. Stimulation parameters were chosen accordingly to induce plasticity in the prefrontal cortex and modulate performance in training tasks, as shown in previous studies of our group, and others (Martin et al., 2013; Meinzer et al., 2013; Wittkuhn et al., 2018).

Multimodal imaging will allow us to examine neural effects through several neural interdependent but also discrete parameters. Resting-state fMRI will allow quantification of functional connectivity in cortical and subcortical networks mediating memory functions such as the fronto-parietal, default mode, and salience network. DTI will allow individual fiber tractography of white matter pathways within these networks and their macro- and microstructural properties. T1-weighted images will allow cortical and subcortical segmentation and volumentry and FLAIR images the identification of white matter hyperintensities. These sequences will determine predictors of response on an individual level, but will also be examined at multiple time points (pre-, post-, and follow-up sessions) in order to scrutinize intervention-induced modulation. In addition, T1and T2-weighted structural sequences will be optimized for the purpose of computational modeling in order to allow accurate simulations of the current flow on an individual basis (Antonenko et al., in press). Blood samples will be collected in order to examine if individual responsiveness to tDCS is modulated by genetic polymorphisms that have previously been shown to modulate neural plasticity and learning ability in several domains (Antal et al., 2010; Freitas et al., 2011; Witte et al., 2012; Plewnia et al., 2013; Stephens et al., 2017). Assessment of daily health conditions, potential pre-session stressors, mood and perceived tDCS-related adverse events will complete the assessment of performance and help to identify potential sources of variability.

Taken together, our Phase IIb clinical trial will provide comprehensive evidence for the effects of a tDCS-plustraining approach in age-associated cognitive decline and

\section{REFERENCES}

Antal, A., Alekseichuk, I., Bikson, M., Brockmoller, J., Brunoni, A. R., Chen, R., et al. (2017). Low intensity transcranial electric stimulation: safety, ethical, legal regulatory and application guidelines. Clin. Neurophysiol. 128, 1774-1809. doi: 10.1016/j.clinph.2017.06.001

Antal, A., Chaieb, L., Moliadze, V., Monte-Silva, K., Poreisz, C., Thirugnanasambandam, N., et al. (2010). Brain-derived neurotrophic factor (BDNF) gene polymorphisms shape cortical plasticity in humans. Brain Stimul. 3, 230-237. doi: 10.1016/j.brs.2009.12.003

Antonenko, D., Hayek, D., Netzband, J., Grittner, U., and Flöel, A. (2019a). tDCSinduced episodic memory enhancement and its association with functional network coupling in older adults. Sci. Rep. 9:2273. doi: 10.1038/s41598-01938630-7

Antonenko, D., Thielscher, A., Saturnino, G. B., Aydin, S., Ittermann, B., Grittner, U., et al. (in press). Towards precise brain stimulation: is electric field simulation related to neuromodulation? Brain Stimul. thus contribute to the understanding of plasticity-inducing interventions in aging, and informing the development of efficient interventions in the future.

\section{TRIAL STATUS}

Recruitment of participants started May 2018. Last follow-up is expected for March 2020.

\section{ETHICS STATEMENT}

The studies involving human participants were reviewed and approved by the ethics committee of University medicine Greifswald. The participants provided their written informed consent to participate in this study.

\section{AUTHOR CONTRIBUTIONS}

DA, S-CL, and AF designed the study and wrote the grant applications. DA, AD, and FraT developed and programmed the cognitive tasks. DA and AF acquired the ethical approval. DA, FriT, and JU prepared the information sheets and study materials. FriT and JU will acquire the data. DA, FriT, and UG will analyze the data. DA, FriT, and AF wrote the study protocol. All authors commented and approved the final version of the manuscript.

\section{FUNDING}

Funding for this study was provided by the "Bundesministerium für Bildung und Forschung” (FKZ 01GQ1424A).

\section{ACKNOWLEDGMENTS}

We would like to thank Dr. Susanne Passow for her assistance in designing the study and Robert Malinowski for programming assistance and technical support.

Antonenko, D., Kulzow, N., Sousa, A., Prehn, K., Grittner, U., and Floel, A. (2018). Neuronal and behavioral effects of multi-day brain stimulation and memory training. Neurobiol. Aging 61, 245-254. doi: 10.1016/j.neurobiolaging.2017. 09.017

Antonenko, D., Schubert, F., Bohm, F., Ittermann, B., Aydin, S., Hayek, D., et al. (2017). tDCS-Induced modulation of GABA levels and resting-state functional connectivity in older adults. J. Neurosci. 37, 4065-4073. doi: 10.1523/ JNEUROSCI.0079-17.2017

Au, J., Karsten, C., Buschkuehl, M., and Jaeggi, S. M. (2017). Optimizing transcranial direct current stimulation protocols to promote long-term learning. J. Cogn. Enhanc. 1, 65-72. doi: 10.1007/s41465-017-0007-6

Bachtiar, V., Johnstone, A., Berrington, A., Lemke, C., Johansen-Berg, H., Emir, U., et al. (2018). Modulating regional motor cortical excitability with noninvasive brain stimulation results in neurochemical changes in bilateral motor cortices. J. Neurosci. 38, 7327-7336. doi: 10.1523/jneurosci.2853-17.2018

Berryhill, M. E. (2017). Longitudinal tDCS: consistency across working memory training studies. AIMS Neurosci. 4, 71-86. doi: 10.3934/Neuroscience.2017.2.71 
Borm, G. F., Fransen, J., and Lemmens, W. A. (2007). A simple sample size formula for analysis of covariance in randomized clinical trials. J. Clin. Epidemiol. 60, 1234-1238. doi: 10.1016/j.jclinepi.2007.02.006

Brink, T. L., Yesavage, J., and Lum, O. (2013). "Geriatric depression scale," in Evidence-Based Diagnosis: A Handbook of Clinical Prediction Rules, ed. M. H. Ebell (Berlin: Springer Science \& Business Media), 297.

Chan, A.-W., Tetzlaff, J. M., Altman, D. G., Laupacis, A., Gøtzsche, P. C., KrležaJerić, K., et al. (2013a). SPIRIT 2013 statement: defining standard protocol items for clinical trials. Ann. Intern. Med. 158, 200-207.

Chan, A.-W., Tetzlaff, J. M., Gøtzsche, P. C., Altman, D. G., Mann, H., Berlin, J. A., et al. (2013b). SPIRIT 2013 explanation and elaboration: guidance for protocols of clinical trials. BMJ 346:e7586. doi: 10.1136/bmj.e7586

Charlton, R. A., Barrick, T. R., Lawes, I. N. C., Markus, H. S., and Morris, R. G. (2010). White matter pathways associated with working memory in normal aging. Cortex 46, 474-489. doi: 10.1016/j.cortex.2009.07.005

Dahlin, E., Neely, A. S., Larsson, A., Backman, L., and Nyberg, L. (2008). Transfer of learning after updating training mediated by the striatum. Science 320, 1510-1512. doi: 10.1126/science. 1155466

Darki, F., and Klingberg, T. (2015). The role of fronto-parietal and fronto-striatal networks in the development of working memory: a longitudinal study. Cereb. Cortex 25, 1587-1595. doi: 10.1093/cercor/bht352

Elmasry, J., Loo, C., and Martin, D. (2015). A systematic review of transcranial electrical stimulation combined with cognitive training. Restor. Neurol. Neurosci. 33, 263-278. doi: 10.3233/rnn-140473

Eppinger, B., Heekeren, H. R., and Li, S. C. (2015). Age-related prefrontal impairments implicate deficient prediction of future reward in older adults. Neurobiol. Aging 36, 2380-2390. doi: 10.1016/j.neurobiolaging.2015.04.010

Faul, F., Erdfelder, E., Lang, A.-G., and Buchner, A. (2007). G* Power 3: a flexible statistical power analysis program for the social, behavioral, and biomedical sciences. Behav. Res. Methods 39, 175-191. doi: 10.3758/bf03193146

Filmer, H. L., Ehrhardt, S. E., Shaw, T. B., Mattingley, J. B., and Dux, P. E. (2019). The efficacy of transcranial direct current stimulation to prefrontal areas is related to underlying cortical morphology. Neuroimage 196, 41-48. doi: 10.1016/j.neuroimage.2019.04.026

Floel, A., and Cohen, L. G. (2010). Recovery of function in humans: cortical stimulation and pharmacological treatments after stroke. Neurobiol. Dis. 37, 243-251. doi: 10.1016/j.nbd.2009.05.027

Formann, A., Waldherr, K., and Piswanger, K. (2011). Wiener Matrizen-Test 2. Manual. Weinheim: Beltz Test.

Freitas, C., Mondragón-Llorca, H., and Pascual-Leone, A. (2011). Noninvasive brain stimulation in Alzheimer's disease: systematic review and perspectives for the future. Exp. Gerontol. 46, 611-627. doi: 10.1016/j.exger.2011.04.001

Fritsch, B., Reis, J., Martinowich, K., Schambra, H. M., Ji, Y., Cohen, L. G., et al. (2010). Direct current stimulation promotes BDNF-dependent synaptic plasticity: potential implications for motor learning. Neuron 66, 198-204. doi: 10.1016/j.neuron.2010.03.035

Hartley, T., Maguire, E. A., Spiers, H. J., and Burgess, N. (2003). The well-worn route and the path less traveled: distinct neural bases of route following and wayfinding in humans. Neuron 37, 877-888. doi: 10.1016/s0896-6273(03) 00095-3

Helmstaedter, C., Lendt, M., and Lux, S. (2001). Verbaler Lern-und Merkfähigkeitstest: VLMT; Manual. Weinheim: Beltz-Test.

Horvath, J. C., Forte, J. D., and Carter, O. (2015). Quantitative review finds no evidence of cognitive effects in healthy populations from single-session transcranial direct current stimulation (tDCS). Brain Stimul. 8, 535-550. doi: 10.1016/j.brs.2015.01.400

Hunter, M. A., Coffman, B. A., Trumbo, M. C., and Clark, V. P. (2013). Tracking the neuroplastic changes associated with transcranial direct current stimulation: a push for multimodal imaging. Front. Hum. Neurosci. 7:495. doi: 10.3389/ fnhum.2013.00495

Jenkinson, M., Beckmann, C. F., Behrens, T. E., Woolrich, M. W., and Smith, S. M. (2012). FSL. Neuroimage 62, 782-790. doi: 10.1016/j.neuroimage.2011.09.015

Jones, K. T., Stephens, J. A., Alam, M., Bikson, M., and Berryhill, M. E. (2015). Longitudinal neurostimulation in older adults improves working memory. PLoS One 10:e0121904. doi: 10.1371/journal.pone.012 1904

Kulzow, N., Cavalcanti de Sousa, A. V., Cesarz, M., Hanke, J. M., Gunsberg, A., Harder, S., et al. (2017). No Effects of non-invasive brain stimulation on multiple sessions of object-location-memory training in healthy older adults. Front. Neurosci. 11:746. doi: 10.3389/fnins.2017.00746

Kuo, M. F., and Nitsche, M. A. (2012). Effects of transcranial electrical stimulation on cognition. Clin. EEG Neurosci. 43, 192-199. doi: 10.1177/1550059412444975

Le Bihan, D., and Johansen-Berg, H. (2012). Diffusion MRI at 25: exploring brain tissue structure and function. Neuroimage 61, 324-341. doi: 10.1016/j. neuroimage.2011.11.006

Lehrl, S. (1977). MWT-B: Mehrfach-Wahl-Wortschatz-Test B. Erlangen: Straube.

Lezak, M. D., Howieson, D. B., Loring, D. W., and Fischer, J. S. (2004). Neuropsychological Assessment. New York, NY: Oxford University Press.

Lindenberg, R., Nachtigall, L., Meinzer, M., Sieg, M. M., and Floel, A. (2013). Differential effects of dual and unihemispheric motor cortex stimulation in older adults. J. Neurosci. 33, 9176-9183. doi: 10.1523/jneurosci.0055-13.2013

Lindenberger, U., and Baltes, P. B. (1997). Intellectual functioning in old and very old age: cross-sectional results from the Berlin aging study. Psychol. Aging 12, 410-432. doi: 10.1037//0882-7974.12.3.410

Mameli, F., Fumagalli, M., Ferrucci, R., and Priori, A. (2014). "Chapter 13 Transcranial Direct Current Stimulation and Cognition in the Elderly A2 Kadosh, Roi Cohen," in The Stimulated Brain, ed. R. C. Kadosh (San Diego, CA: Academic Press), 371-395. doi: 10.1016/b978-0-12-404704-4.00013-2

Martin, D. M., Liu, R., Alonzo, A., Green, M., Player, M. J., Sachdev, P., et al. (2013). Can transcranial direct current stimulation enhance outcomes from cognitive training? A randomized controlled trial in healthy participants. Int. J. Neuropsychopharmacol. 16, 1927-1936. doi: 10.1017/s1461145713000539

Meinzer, M., Antonenko, D., Lindenberg, R., Hetzer, S., Ulm, L., Avirame, K., et al. (2012). Electrical brain stimulation improves cognitive performance by modulating functional connectivity and task-specific activation. J Neurosci. 32, 1859-1866. doi: 10.1523/JNEUROSCI.4812-11.2012

Meinzer, M., Lindenberg, R., Antonenko, D., Flaisch, T., and Floel, A. (2013). Anodal transcranial direct current stimulation temporarily reverses ageassociated cognitive decline and functional brain activity changes. J. Neurosci. 33, 12470-12478. doi: 10.1523/jneurosci.5743-12.2013

Metzler-Baddeley, C., Foley, S., de Santis, S., Charron, C., Hampshire, A., Caeyenberghs, K. (2017). Dynamics of white matter plasticity underlying working memory training: multimodal evidence from diffusion MRI and relaxometry. J. Cogn. Neurosci 31, 1509-1520. doi: 10.1162/jocn_a_01127

Metzler-Baddeley, C., Jones, D. K., Belaroussi, B., Aggleton, J. P., and O’Sullivan, M. J. (2011). Frontotemporal connections in episodic memory and aging: a diffusion MRI tractography study. J. Neurosci. 31, 13236-13245. doi: 10.1523/ JNEUROSCI.2317-11.2011

Morris, J., Heyman, A., Mohs, R., Hughes, J., Van Belle, G., Fillenbaum, G., et al. (1989). The consortium to establish a registry for Alzheimer's disease (CERAD): I. Clinical and neuropsychological assessment of Alzheimer's disease. Neurology 39, 1159-1165.

Nilsson, J., Lebedev, A. V., Rydstrom, A., and Lovden, M. (2017). Direct-current stimulation does little to improve the outcome of working memory training in older adults. Psychol. Sci. 28, 907-920. doi: 10.1177/0956797617698139

Oldfield, R. C. (1971). The assessment and analysis of handedness: the Edinburgh inventory. Neuropsychologia 9, 97-113. doi: 10.1016/0028-3932(71)90067-4

Park, S.-H., Seo, J.-H., Kim, Y.-H., and Ko, M.-H. (2014). Long-term effects of transcranial direct current stimulation combined with computer-assisted cognitive training in healthy older adults. Neuroreport 25, 122-126. doi: 10 . 1097/WNR.0000000000000080

Passow, S., Thurm, F., and Li, S. C. (2017). Activating developmental reserve capacity via cognitive training or non-invasive brain stimulation: potentials for promoting fronto-parietal and hippocampal-striatal network functions in old age. Front. Aging Neurosci. 9:33. doi: 10.3389/fnagi.2017.00033

Perceval, G., Floel, A., and Meinzer, M. (2016). Can transcranial direct current stimulation counteract age-associated functional impairment? Neurosci. Biobehav. Rev. 65, 157-172. doi: 10.1016/j.neubiorev.2016.03.028

Plewnia, C., Zwissler, B., Längst, I., Maurer, B., Giel, K., and Krüger, R. (2013). Effects of transcranial direct current stimulation (tDCS) on executive functions: influence of COMT Val/Met polymorphism. Cortex 49, 1801-1807. doi: 10. 1016/j.cortex.2012.11.002

Reis, J., Schambra, H. M., Cohen, L. G., Buch, E. R., Fritsch, B., Zarahn, E., et al. (2009). Noninvasive cortical stimulation enhances motor skill acquisition over multiple days through an effect on consolidation. Proc. Natl. Acad. Sci. U.S.A. 106, 1590-1595. doi: 10.1073/pnas.0805413106 
Schlaug, G., and Renga, V. (2008). Transcranial direct current stimulation: a noninvasive tool to facilitate stroke recovery. Expert Rev. Med. Devices 5, 759-768. doi: 10.1586/17434440.5.6.759

Stephens, J. A., and Berryhill, M. E. (2016). Older adults improve on everyday tasks after working memory training and neurostimulation. Brain Stimul. 9, 553-559. doi: 10.1016/j.brs.2016.04.001

Stephens, J. A., Jones, K. T., and Berryhill, M. E. (2017). Task demands, tDCS intensity, and the COMT val(158)met polymorphism impact tDCS-linked working memory training gains. Sci. Rep. 7:13463. doi: 10.1038/s41598-01714030- 7

Summers, J. J., Kang, N., and Cauraugh, J. H. (2016). Does transcranial direct current stimulation enhance cognitive and motor functions in the ageing brain? A systematic review and meta- analysis. Ageing Res. Rev. 25, 42-54. doi: 10.1016/ j.arr.2015.11.004

Tanaka, S. C., Doya, K., Okada, G., Ueda, K., Okamoto, Y., and Yamawaki, S. (2016). "Prediction of immediate and future rewards differentially recruits cortico-basal ganglia loops," in Behavioral Economics of Preferences, Choices, and Happiness, eds S. Ikeda, H. K. Kato, F. Ohtake, and Y. Tsutsui (New York, NY: Springer), 593-616. doi: 10.1007/978-4-431-55402-8_22

Thielscher, A., Antunes, A., and Saturnino, G. B. (2015). "Field modeling for transcranial magnetic stimulation: a(useful)tool to understand the physiological effects of TMS?", in Proceedings of the 2015 37th Annual International Conference of the IEEE Engineering in Medicine and Biology Society (EMBC), (Milan: IEEE), 222-225.

United Nations, (2015). World Population Ageing. New York, NY: Department of Economic and Social Affairs, Population Division.

Watson, D., Clark, L. A., and Tellegen, A. (1988). Development and validation of brief measures of positive and negative affect: the PANAS scales. J. Pers. Soc. Psychol. 54, 1063-1070. doi: 10.1037//0022-3514.54.6. 1063

Whitfield-Gabrieli, S., and Nieto-Castanon, A. (2012). Conn: a functional connectivity toolbox for correlated and anticorrelated brain networks. Brain Connect. 2, 125-141. doi: 10.1089/brain.2012.0073

Windhoff, M., Opitz, A., and Thielscher, A. (2013). Electric field calculations in brain stimulation based on finite elements: an optimized processing pipeline for the generation and usage of accurate individual head models. Hum. Brain Mapp. 34, 923-935. doi: 10.1002/hbm.21479

Witte, A. V., Kürten, J., Jansen, S., Schirmacher, A., Brand, E., Sommer, J., et al. (2012). Interaction of BDNF and COMT polymorphisms on paired-associative stimulation-induced cortical plasticity. J. Neurosci. 32, 4553-4561. doi: 10.1523/ JNEUROSCI.6010-11.2012

Wittkuhn, L., Eppinger, B., Bartsch, L. M., Thurm, F., Korb, F. M., and Li, S. C. (2018). Repetitive transcranial magnetic stimulation over dorsolateral prefrontal cortex modulates value-based learning during sequential decision-making. Neuroimage 167, 384-395. doi: 10.1016/j.neuroimage.2017. 11.057

Woods, A. J., Antal, A., Bikson, M., Boggio, P. S., Brunoni, A. R., Celnik, P., et al. (2016). A technical guide to tDCS, and related non-invasive brain stimulation tools. Clin. Neurophysiol. 127, 1031-1048. doi: 10.1016/j.clinph.2015. 11.012

Woods, A. J., Cohen, R. A., and Pahor, M. (2013). Cognitive frailty: frontiers and challenges. J. Nutr. Health Aging 17, 741-743. doi: 10.1007/s12603-013-0398-8

Yam, A., Gross, A. L., Prindle, J. J., and Marsiske, M. (2014). Ten-year longitudinal trajectories of older adults' basic and everyday cognitive abilities. Neuropsychology 28, 819-828. doi: 10.1037/neu0000096

Yam, A., and Marsiske, M. (2013). Cognitive longitudinal predictors of older adults' self-reported IADL function. J. Aging Health 25(8 Suppl.), 163S-185S. doi: $10.1177 / 0898264313495560$

Conflict of Interest Statement: The authors declare that the research was conducted in the absence of any commercial or financial relationships that could be construed as a potential conflict of interest.

Copyright (c) 2019 Antonenko, Thams, Uhrich, Dix, Thurm, Li, Grittner and Flöel. This is an open-access article distributed under the terms of the Creative Commons Attribution License (CC BY). The use, distribution or reproduction in other forums is permitted, provided the original author(s) and the copyright owner(s) are credited and that the original publication in this journal is cited, in accordance with accepted academic practice. No use, distribution or reproduction is permitted which does not comply with these terms. 